\title{
Realization of 4G Downlink Channel
}

\author{
Nigidita Pradhan ${ }^{1 *}$, Rabindranath Bera ${ }^{2}$, Debasish Bhaskar ${ }^{3}$ and Preman Chettri ${ }^{4}$ \\ ${ }^{1} P G$ student [DE\&C], Dept. of ECE, Sikkim Manipal Institute of Technology, \\ India \\ ${ }^{2}$ Head of the Dept [HOD], Dept. of ECE, Sikkim Manipal Institute of Technology, \\ India \\ ${ }^{3}$ Assistant Professor, Dept. of ECE, Sikkim Manipal Institute of Technology, India \\ ${ }^{4}$ Junior Engineer, Dept. of ECE, Sikkim Manipal Institute of Technology, India, \\ Majitar, Rangpo, East Sikkim-737136, India \\ Niki.pradhan92@gmail.com
}

\begin{abstract}
Today's era people have grown in every field, such that their needs are also growing linearly with their advancement. This rapid development of broadband wireless access technologies in recent years was the result of growing demand for mobile Internet and wireless multimedia applications. Now it is becoming difficult to meet the demands of these data hungry people. So, the wireless network is advancing after $3 G$ and providing better coverage, higher data rate and by reducing Latency. It uses the frame structure as Time Division Duplexing (TDD) and Frequency Division Duplexing (FDD).LTE technology uses different channels in both uplink and downlink. This paper mainly focuses on the Downlink channels named as PDCCH, PBCH, PCFICH, PHICH and downlink Signals i.e., PSS and S-SS. The individual Downlink Channels are analyzed using Agilent SystemVue platform further it is synchronized with the Agilent Vector Signal Analyzer (VSA) to the check the Output Response of the individual downlink channels.
\end{abstract}

Keywords: PCFICH, PDCCH, PHICH, PBCH, P-SS, S-SS, LTE, 3GPP, TDD, FDD, CCEs and REGs

\section{Introduction}

4G, short for Fourth Generation, is the successor of the today's existing 3G technology. And is deployed by $3^{\text {rd }}$ Generation. Two groups, 3GPP (Third Generation Partnership Project) and 3GPP2 (Third Generation Partnership Project 2) are working together to form the foundation for LTE, where 3GPP represents the functions of GSM family and latter represents the functions of CDMA family .4G technologies are designed to provide IP-based voice, data and Multimedia streaming at the speed of atleast $100 \mathrm{Mbit} / \mathrm{sec}$. With the exception, the existing mobile networks have until recently been relying purely on 3G/UMTS technology, which is 10 yrs old and struggling to cope with the needs of today's data hungry users[1]. The $4 \mathrm{G}$ is a new generation of wireless that replaces the $3 \mathrm{G}$ systems. The key features of the $4 \mathrm{G}$ infrastructures are accessing information anywhere, anytime, with a seamless connection to a wide range of information and services, and receiving a large volume of information, data, pictures, video, and so on. Based on the developing trends of mobile communications, the $4 \mathrm{G}$ focus on ensuring seamless service across a multitude of wireless systems and networks and have broader bandwidth, higher data rate, and smoother and quicker handoff. LTE downlink channels are PCFICH, $\mathrm{PDCCH}, \mathrm{PMCH}$ and PBCH. Among these, PHICH, PCFICH and PDCCH are coming under the classification of downlink control channels. Excluding these downlink control channels, rest is the downlink data channels [2]. In this paper the overview of the $4 \mathrm{G}$ will be discussed. Signal Measurements within the system reflect the healthy condition of any 
electronics system like 4G_LTE. Signal testing at different levels like during design, testing at the production level, testing during repair even at the initial phase of understanding of the system are helpful. Therefore, for better understanding of 4G_LTE, this paper will enlighten Signal Measurement of downlink channels which helps to understand the DESIGN and SYSTHESIS of the 4G mobile system. The platform used here is the Agilent SystemVue Software. This is synchronized with the Agilent Vector Signal Analyzer (VSA) to visualize the output waveforms.

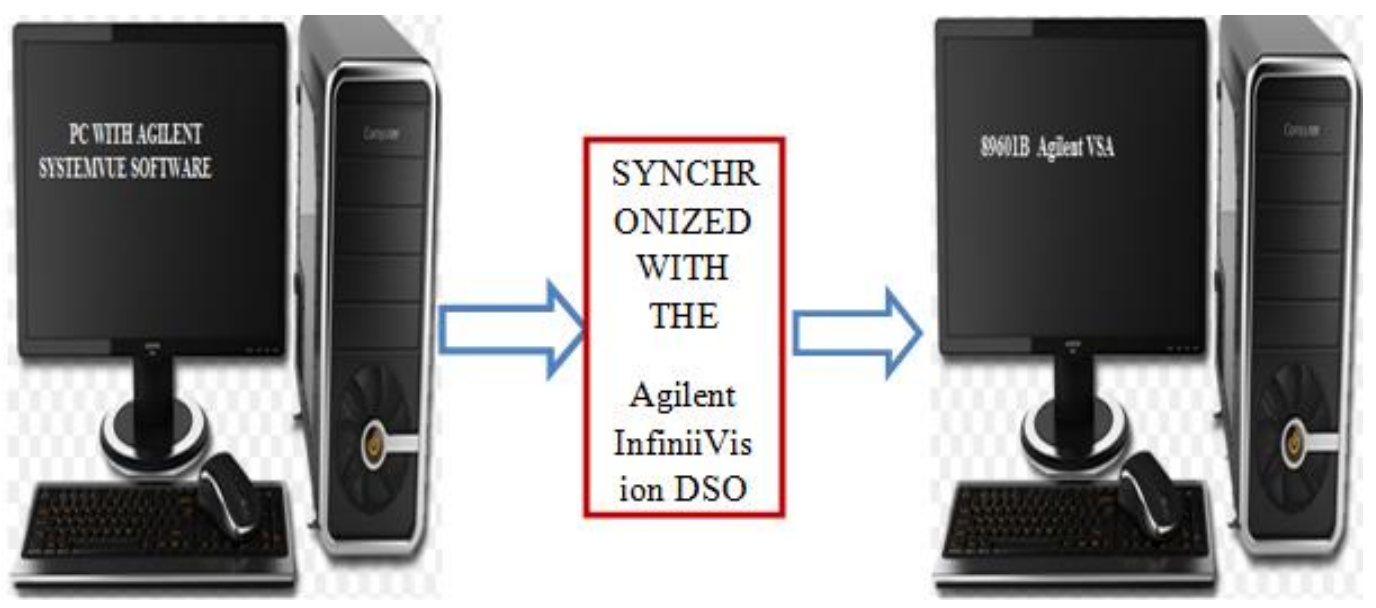

Figure 1. Basic Block Diagram

The above Figure 1 Shows the basic block diagram. In which the Pc with Agilent SystemVue software is connected with the Agilent InfiniiVision DSO (7032B) hardware and using LAN the DSO is synchronized with another PC with Agilent VSA software. So the data from the oscilloscope is fetched to the System with VSA software.

\section{Literature Survey}

Wireless network has been improved after $3 \mathrm{G}$ by means of higher data rates with better coverage and by reducing latency. The main objective of this paper is the realization of the SISO (Single Input- Single Output) architecture of PBCH downlink data channel with its own transmitter and receiver. Information is processed at transmitter by means of scrambling, modulation, layer mapping, precoding and mapping to resource elements. Similar to the transmitter, receiver also has to perform some techniques to retrieve the original data such as demapping from resource elements, decoding, delayer mapping, demodulation and descrambling. Simulation of these steps for transmitter is done by using ModelSim 6.4a and the synthesis and implementation are done by using PlanAhead 13.2 virtex-5, xc5vlx50tff1136-1 board. Power estimation, resource estimation, RTL designs and FPGA editors are shown for the transmitter and receiver structure [1].

This paper presented a brief description of path to $4 \mathrm{G}$ networks, WiMAX and LTE Network architecture and OFDMA technology. It has been observed that the number of wireless broadband subscribers have passed the number of fixed broadband subscribers. So in a world going wireless, the technologies with higher throughputs get importance day by day [4]. This paper describes the applications of $4 \mathrm{G}$ technology considering the importance of switching to $4 \mathrm{G}$ systems as a better service compared to the $3 \mathrm{G}$ technology the author presents both external and internal diversity of each target to illustrate the causes and solutions of the adaptability feature. Then, the $4 \mathrm{G}$ domain of each feature in the framework is discussed from technical point, showing techniques and possible research issues for sufficient support of adaptability. At the end, a summary on $4 \mathrm{G}$ visions and some of the issues this new technology may face [5]. 
Orthogonal Frequency Division multiplexing (OFDM) has been recognized as one of the most promising techniques to meet these challenges. Space Time block Coding (STBC) has drawn much attention in $4 \mathrm{G}$ wireless technologies just because of its decoding simplicity. This paper presents the performance evaluation of STBC-OFDM systems covering channel model and coding scheme, it includes Rayleigh fading channels by which it is observed that Bit Error Rate (BER) is reduced to achieve the high data rate [7].

Mobile communication system can cause serious performance degradation on the cell edge. This problem is essentially due to non-uniform distribution of the radio power of the cellular system. It can be softened to the certain extent by using new radio Architecture called Distributed Antenna System (DAS).The main objective of the author is to tackle the shortcomings due to the cell edge effect in cellular system by suitably changing the existing Collocated Antenna System (CAS) to a distributed Antenna System (DAS) and to compare its performance in terms of Bit Error Rate (BER) and capacity [9].

The author discuss the improvement of the Technologies from $1 \mathrm{G}$ to the current, also the author discussed the approaches/methodologies for upgradation how it can be further improved for the Future. This paper has also covered the standards and also the Architecture of the Future Generation [10].

\section{Frame Mode}

Frame Mode of LTE (Long Term Evolution), the type of enum and it can be selected as FDD and TDD.. FDD supports Frame structure Type-I and TDD supports frame structure Type-II.

\section{A. Frame Structure}

\section{Type I (FDD) mode}

The Type 1FDD mode employs a different frame structure depending on whether the Transmission is downlink or uplink. For either link direction, however a radio frame has duration of $10 \mathrm{~ms}$ consist of 20 slots with slot duration of $0.5 \mathrm{~ms}$. Two slots comprise a sub-frame. A subframe also known as the transmission time interval (TTI has a duration) of $1 \mathrm{~ms}$.

1 Frame $=10$ Sub-Frame $=10 \mathrm{~ms}$

1 Subframe $=2$ slots $=1 \mathrm{~ms}$ (one slots $=0.5 \mathrm{~ms}$ )

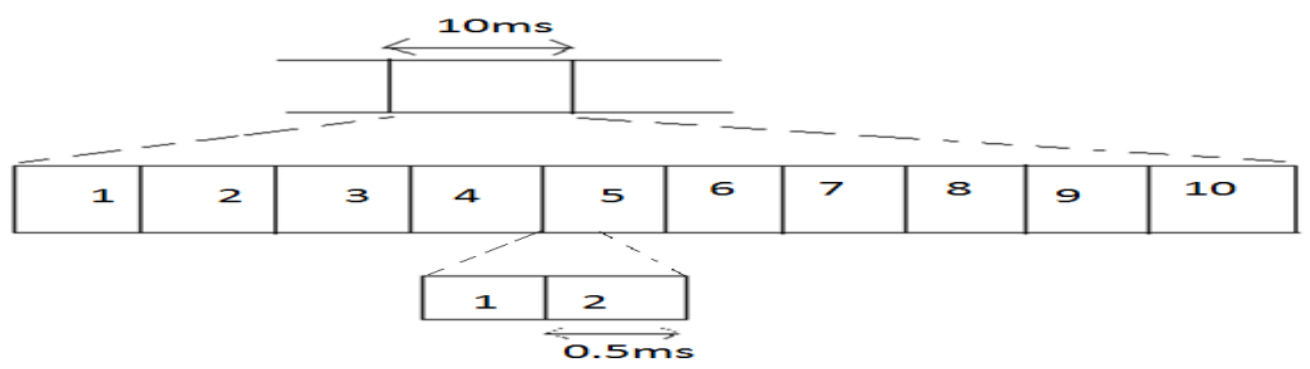

Figure 1.1. Frame FDD Mode

Subcarrier BW $=15 \mathrm{KHz}$

Maximum rate of FFT and IFFT $=2048$

Sampling Time $\left(\mathrm{T}_{\mathrm{s}}\right)=1 / \mathrm{Max}^{\mathrm{m}}$ rate of IFFT $\mathrm{x}$ Subcarrier Bandwidth

$$
\frac{1}{2048 * 15 * 10^{3}}=3.255 \times 10^{-8}
$$

$=32.6 \mathrm{nsec}$

Sampling Frequency $\left(F_{s}\right)=1 / T_{s}$ 


$$
\begin{aligned}
& =2048 \times 15 \times 10^{3} \\
& =30.72 \mathrm{MHz}
\end{aligned}
$$

In time domain, Every Frame Structure has to be represented in terms of $T_{s}$

$\mathrm{T}_{\text {frame }}=$ Normalized Factor $\mathrm{x} \mathrm{T}_{\mathrm{s}}$

$=30.72 \times 10^{6} \times 32.6 \times 10^{-9}$

$=0.01 .14$

$=10 \times 10^{-3}=10 \mathrm{msec}$

\section{- BANDWIDTH}

Long term Evolution (LTE) supports the Bandwidth 1.4MHz, BW $3 \mathrm{MHz}$, BW 5MHz, BW $10 \mathrm{MHz}$, and BW 15MHz and BW $20 \mathrm{MHz}$. bandwidth can be varied using Resource Block.

\section{- OFDMA}

OFDMA or Orthogonal Frequency -division multiplexing which is also known as Discrete Multitone Modulation (DMT), is a modulation method for the modulation of a frequency channel based on the FDM(frequency division multiplexing) .In this technique, the frequencies and modulations of frequency division multiplexing are arranged orthogonally to each other to eliminate any interference between the channels. The main aim of OFDMA is to send the low rate modulations in a parallel stream rather than sending a high rate wave front, as low rate modulations are less sensitive to multipath. With OFDM technology already proved and embraced, 4G development will gain momentum [1].

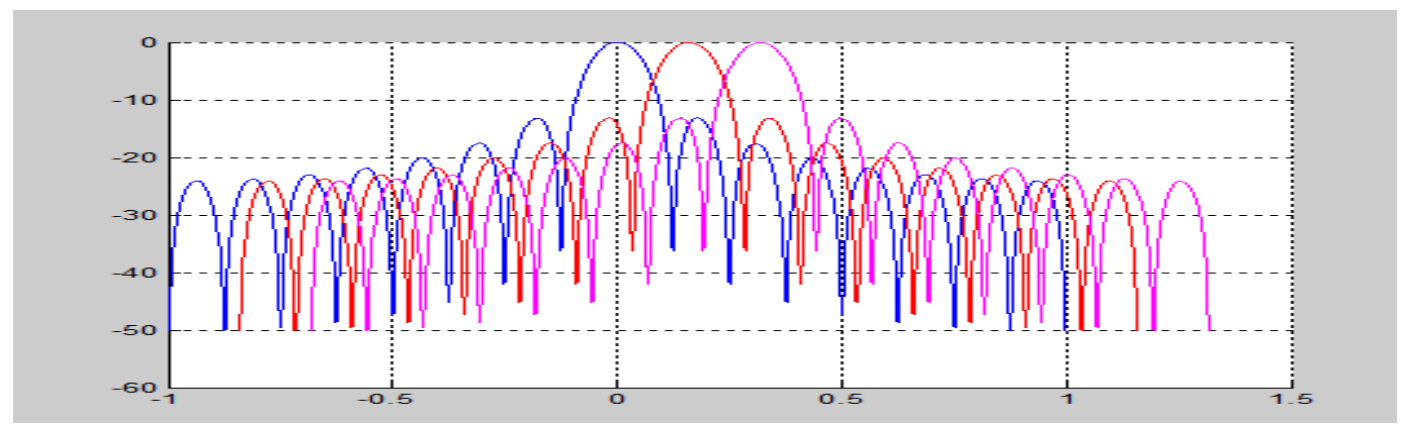

Figure 2. Orthogonal Frequency Division Multiplexing

The LTE downlink is composed of two sets of Physical Layer channel.

- Physical channels

- Physical Signals

The Physical channels are Physical Downlink Control Channel (PDCCH), Physical downlink Control Format Indicator Channel (PCFICH) and Physical Broadcast Channel $(\mathrm{PBCH})$. And the Physical Signals are Refrence Signals (RS) and Synchronization Signals i.e., Primary Synchronization Signals (P-SS) and Secondary Synchronization Signals (S$\mathrm{SS})$.

\section{Downlink Physical Channels}

\section{a. Physical Broadcast Channel (PBCH)}

It carries the Broadcast Channel (BCH) transport Channel. It supports QPSK Modulation. The BCH carries various cell-specific content and is used for all type of UE.As with the Synchronization signals the $\mathrm{PBCH}$ is transmitted in the centre of the channel but it occupies $6 \mathrm{RB}$ (72 Subcarrier). This is the whole of the narrowest channel 
Bandwidth. The PBCH broadcasts a limited number of parameters essential for initial access of the cell such as downlink system bandwidth, the Physical Hybrid ARQ Indicator Channel structure, and the most significant eight-bits of the System Frame Number [2].

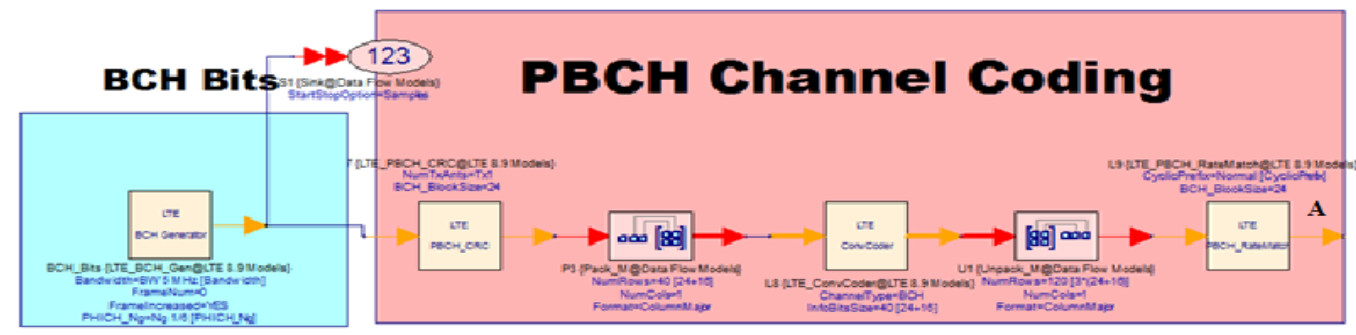

\section{Figure 3a. PBCH Bits Generation and Channel Coding in the Tx Section}

The first block is the block is the BCH generator. It generates 24 bits of Master Information Bits. Then the Second block is CRC which generates the 16 bits of CRC and it is scrambled with a antenna specific mask. The Generated CRC is attached to the MIB payload after which the size of the Payload will be 40 bits (24bitof MIB+16 bit of CRC). A tail bit convolution encoding is performed over the 40 bits and the output is 3 streams of 40 bits each. The rate matching here is nothing but a repetition coding where the 3 streams of size 120 bits (40x3bits) is just repeated 16 times to get 1920 bits. The repetition rate is very high since the MIB is a very vital and UE cannot afford to lose it.

\section{Scrambling and Modulation}

L10 \{LTE_PBCH_Scrambler@LTE 8.9 Models\}

Cyclicerefix $=$ Normal
CelliD_Sector $=0$ [CelliD_Sector]

CellID_Group $=0$ [CellID_Group]
Cons

A

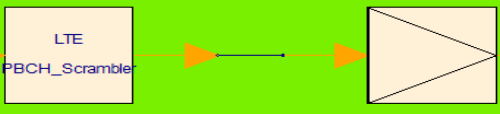

B9 \{BitsToInt@DataFlow Models\}

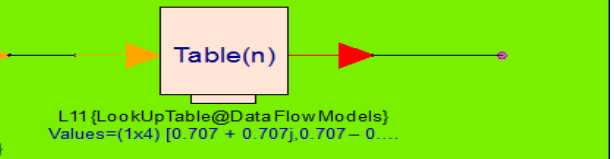

Figure 3b. PBCH Scrambling and Modulation Section in the TX Section

In the Scrambling block these 1920 bits are scrambled with a scrambling sequence as long as 1920 bits. Then a QPSK modulation is performed over these 1920 bits to obtain 960 complex QPSK Symbols.

\section{b. Physical Control Format Indicator Channel}

PCFICH conveys the number of control symbols in the current subframe. Since a subframe in the LTE FDD can have upto 14 symbols for normal cp scenario, among the 14 OFDM symbols there can be upto 3 control symbols depending upon the amount of control data the eNode has to transmit. The number of control symbols can be 1,2 or 3 . It can also be 4 in case of lower bandwidth system such as $3 \mathrm{MHz}$. 


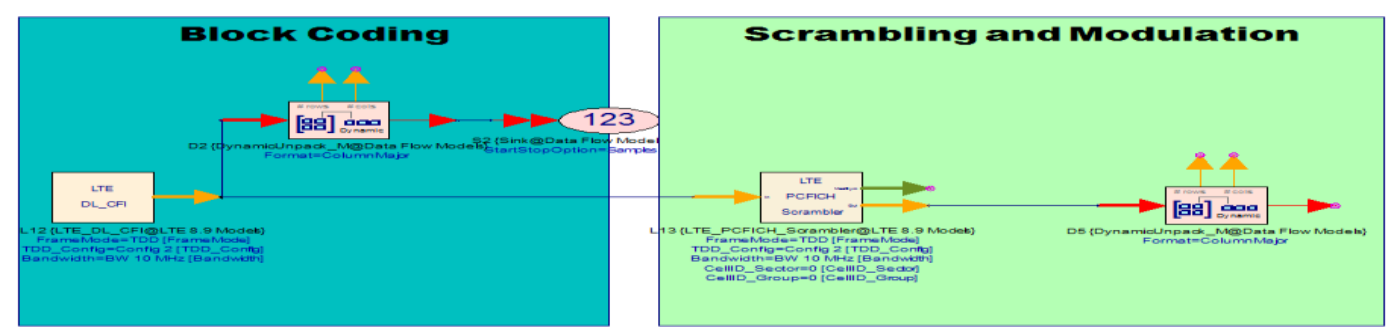

Figure 3c. PCFICH TX Section

Block Coding Standard has 32 bit codeword corresponding to each CFI value. It's a simple Look up encoding, where the CFI is $1 / 32$ rate encoded, which means the input to the Block coding Stage is CFI value and the Output is 32 bit codeword. In the Scrambling block a 32 bit length scrambling sequence is generated using slot number cell ID. The generated scrambling sequence is XORed with 32 codeword's generated in previous stage. Then the QPSK modulation is performed over 32 codeword and since QPSK takes 2 bit per symbol, the output of this stage is QPSK symbols.

\section{c. Physical Downlink Control Channel}

A PDCCH is transmitted on one or an aggregation of several consecutive control channel elements (CCEs). A CCE is a group of nine consecutive Resource-Element Groups (REGs). The number of CCEs used to carry a PDDCH is controlled by PDCCH format. A PDCCH format of $0,1,2$ or 3 corresponds to 1, 2, 3 or 4 consecutive CCEs being allocated to one PDCCH.

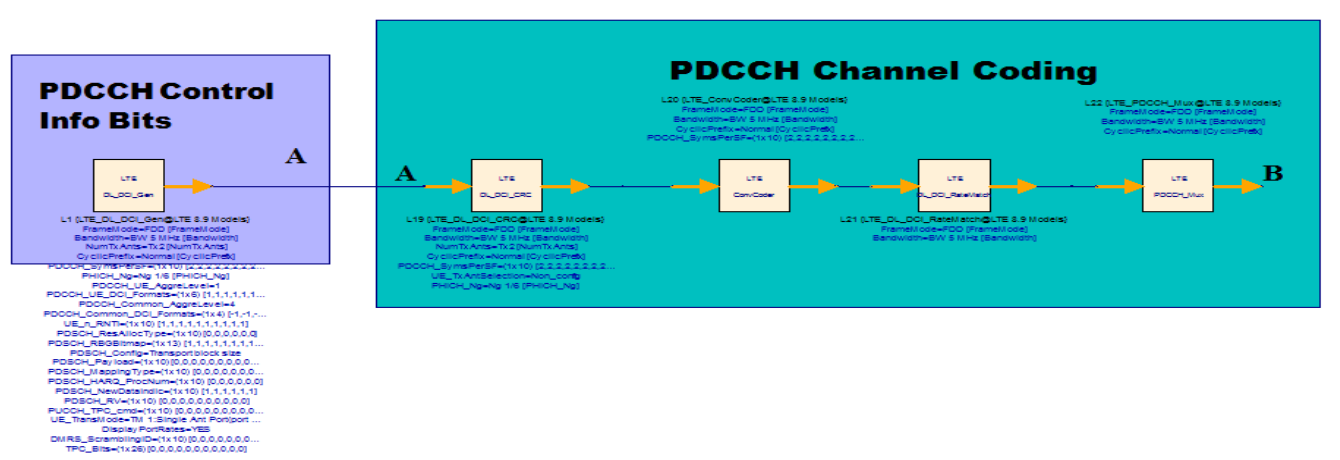

Figure 3d. PDCCH Control Info Bits and Channel Coding

The DCI message with the CRC attachment undergoes tail biting convolution coding. Convolution coding is a form of forward error correction and improves the channel capacity by adding carefully selected redundant information. LTE uses a rate $1 / 3$ tailbiting encoder with a constraint length, $\mathrm{k}$, of 7 . This means that one in three bits of the output contain useful information while the other two add Redundancy. The tail biting convolution encoder initializes its internal shift register to last $\mathrm{k}$ bits of the current input block, rather than to all zeros state. Thus, the start and the end states are the same, without the need to zero-pad the input block. Since the overhead of terminating the coder has been eliminated, the output block contains fewer bits than a standard convolution coder. The drawback is that the decoder becomes more complicated because the initial state is unknown; however, the decoder does know the start and end states are same. The Rate Matching block creates an output bit stream with a desired code rate. As the number of bits available for Transmission depends on the Available resources the rate matching algorithm is capable of producing any arbitrary rate. The three bit streams from the turbo encoder are interleaved followed by bit interleaved collection to create a circular buffer. Bits are selected and pruned from the buffer to create an output bit stream with the desired 
code Rate. The blocks of coded bits for each control channel are multiplexed in order to create a block of data.

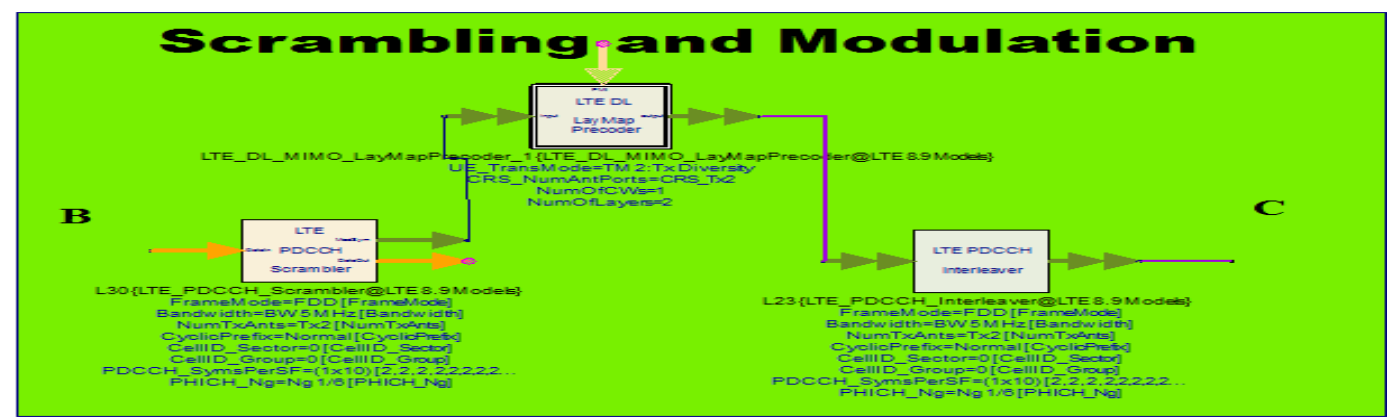

Figure 3e. PDCCH Scrambling and Modulation

This Multiplexed Block of bits undergoes a bit-wise exclusive- or (XOR) operation with a cell-specific scrambling sequence. The scrambling sequence is pseudorandom, created using a length-31 Gold sequence generator and initialized using a slot number within the radio Frame, $\mathrm{n}_{\mathrm{s}}$, and the cell ID, $\mathrm{N}_{\mathrm{ID}}{ }^{\text {cell }}$ at the start of each subframe, as shown in the equation below.

$$
\mathrm{C}_{\text {init }}=\left[\frac{\mathrm{ns}}{2}\right] 2^{9}+\mathrm{N}_{\mathrm{ID}}{ }^{\text {cell }}
$$

Scrambling serves the purpose of intercell interference rejection. When a UE descrambles a received bits stream with a known cell specific scrambling sequence, interference from other cells will be descrambled incorrectly, therefore only appearing as uncorrelated symbols. The Scrambled bits then undergoes QPSK modulation to create a block of complex-valued modulation symbols [2].

\section{d. Physical Hybrid-ARQ Indicator Channel}

The Physical Hybrid-ARQ Indicator Channel (PHICH) in the downlink carries Hybrid ARQ (HARQ) acknowledgements (ACK/NACK) for uplink data transfers. PHICHs are located in the first OFDM symbol of each subframe. A PHICH is carried by several Resource Element Groups (REGs). Multiple PHICHs can share the same set of REGs and are differentiated by orthogonal covers; $\mathrm{PHICHs}$ which share the same resources are called a PHICH group. Consequently, a specific PHICH is identified by two parameters: the PHICH group number, and the orthogonal sequence index within the group.

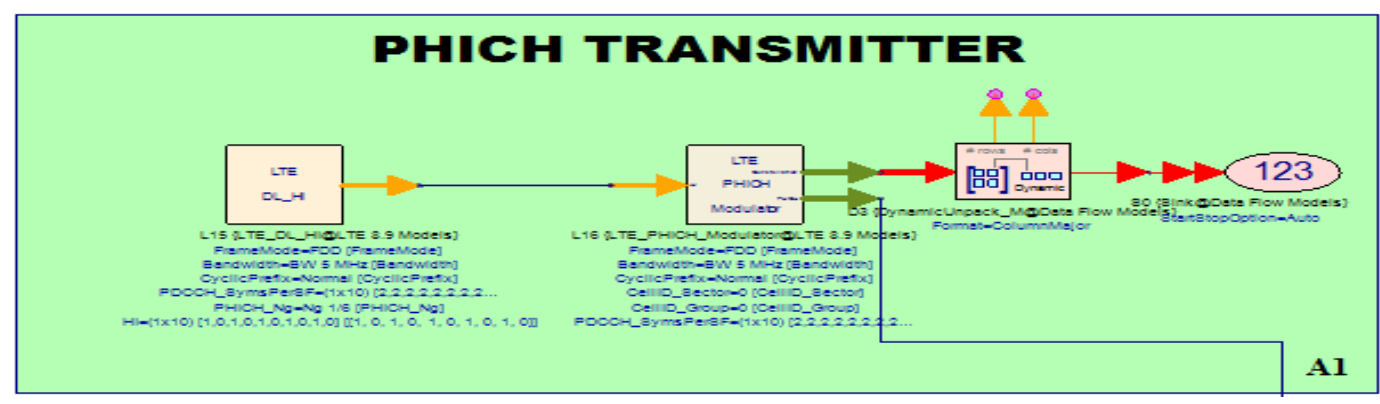

Figure 3f. PHICH Transmitter

The HARQ Indicator Codeword undergoes BPSK modulation, Scrambling, Layer mapping, precoding, and resource mapping. 


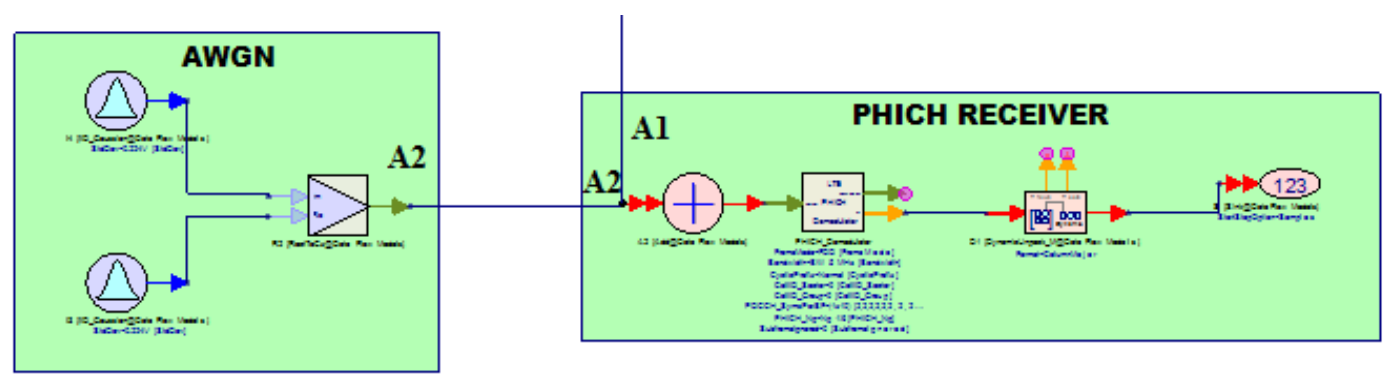

Figure 3g. Addition of Noise

The HARQ indicator codeword undergoes BPSK modulation resulting in a block of complex-valued modulated symbols, $z(0), z(1), z(2)$. The block of modulated symbols is bitwise multiplied with an orthogonal sequence and a cell-specific scrambling sequence to create a sequence of symbols, $\mathrm{d}(0), \ldots, \mathrm{d}\left(\mathrm{M}_{\mathrm{symb}}-1\right)$. The number of symbols, $M_{\text {symb }}$, is given by the equation $\mathrm{M}_{\text {Symb }}=3 \times \mathrm{N}_{\mathrm{PHICHSF}}$. The $\mathrm{PHICH}$ spreading factor, NPHICHSF, is 4 for a normal cyclic prefix and 2 for an extended cyclic prefix. The orthogonal sequence allows multiple PHICHs to be mapped to the same set of resource elements. Scrambling with a cell-specific sequence serves the purpose of intercell interference rejection. When a UE descrambles a received bit stream with a known cell specific scrambling sequence, interference from other cells will be descrambled incorrectly and therefore only appear as uncorrelated noise.

\section{Preliminary Results}

4G_LTE Signal measurements using Keysight VSA (Vector Signal Analyzer)

\section{* Synchronization Signal}

It consist of two 31-bit BPSK M-sequence, and is used for cell search and identification by UE. Carries the Remainder of cell ID (one of the 168 binary sequences). The below given is the Agilent Vector Signal Analyzer signal (VSA) for S-SS.

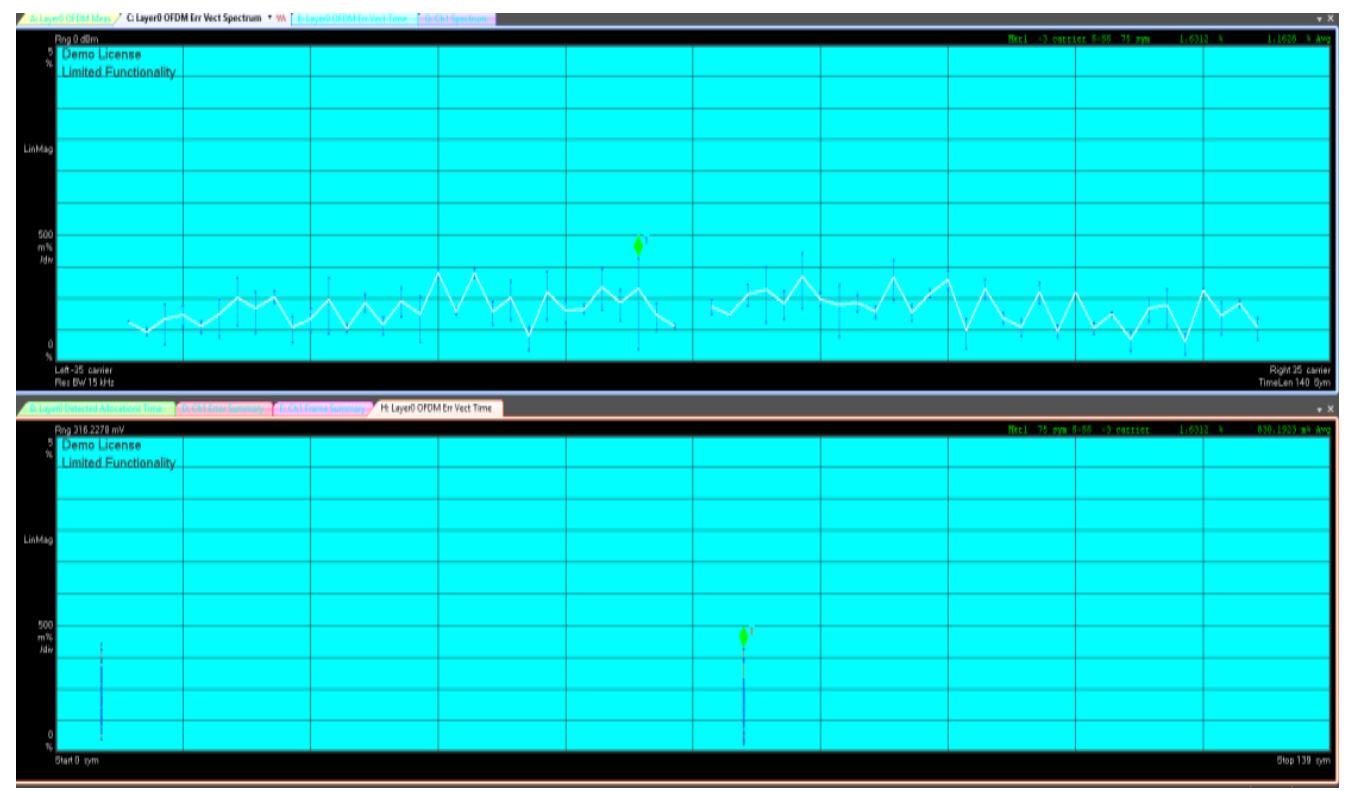

Figure 4a. Secondary Synchronization Signal 


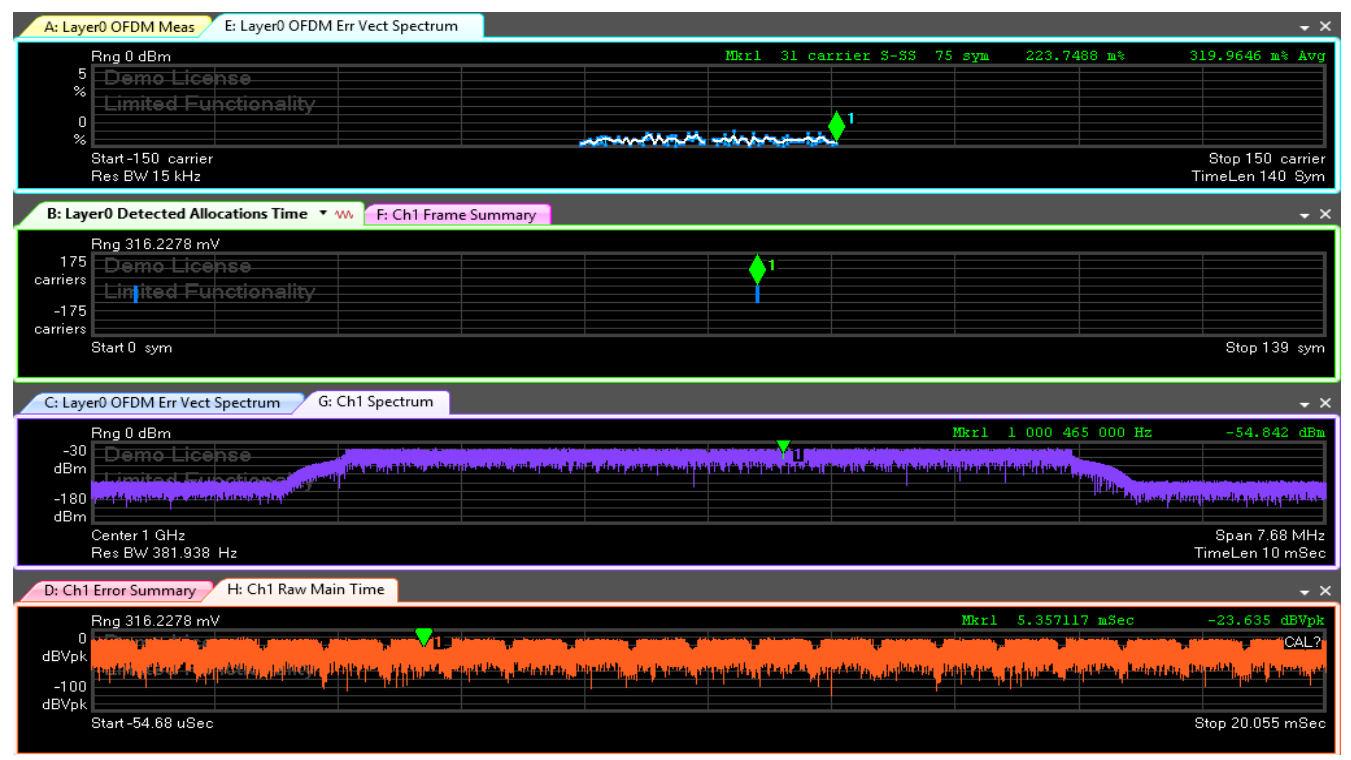

Figure 4b. Secondary Synchronization Signal

Layer0_OFDM_Error_Vector_Spectrum: Only subcarrier indices are visible (i.e., -31 to 31). No actual value of subcarrier is known.Layer0_Detected_Allocations_Time: Symbol index can be known from this plot. But actual time value can't be known for any S-SS symbol. So, to avoid this problem, the following measurement traces are explored in VSA 89600B software.Ch1_Spectrum: The actual spectrum width is $0.93 \mathrm{MHz}$. Ch1_Raw_Main_Time: The 1st S-SS Symbol occurs at 357.117464 microsec and 2nd SSS Symbol occurs at $5.357117 \mathrm{~ms}$.

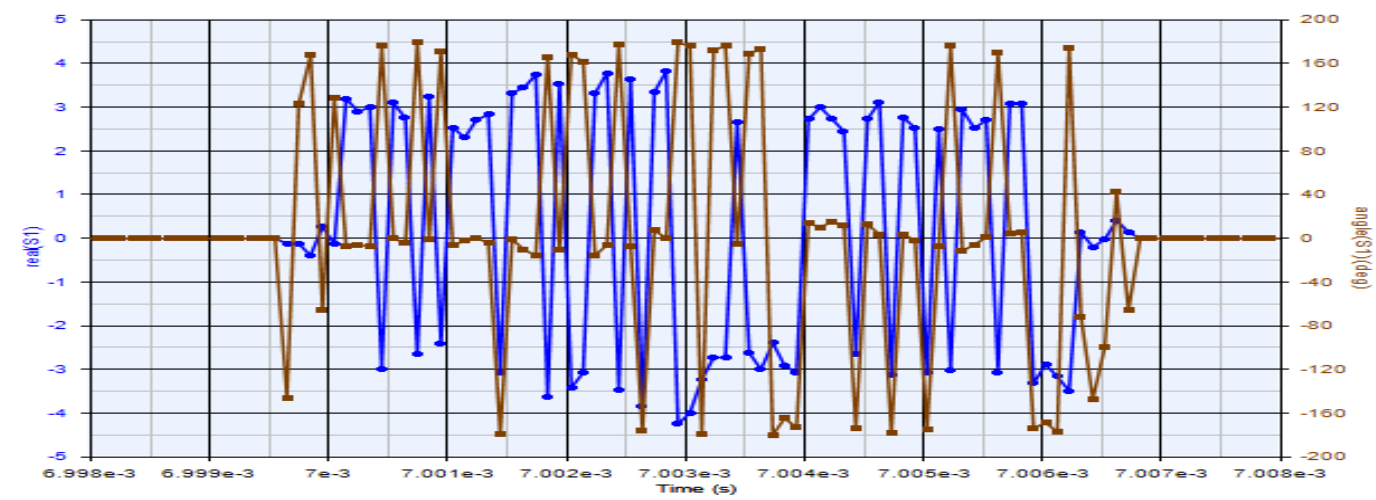

Figure 4c. S-SS Symbols with BPSK Modulation (Blue - Real Part of the SSS Mod symbols and Brown-Phase plot of the S-SS Mod symbols)

\section{* Primary Synchronization Signal}

It consists of one of the 3 Zadoff-Chu sequences and is used for cell search and identification by the UE. Carriers part of the cell ID (one of 3 orthogonal sequences). The Below given is the Agilent Vector Signal Analyzer signal (VSA) for P-SS. 


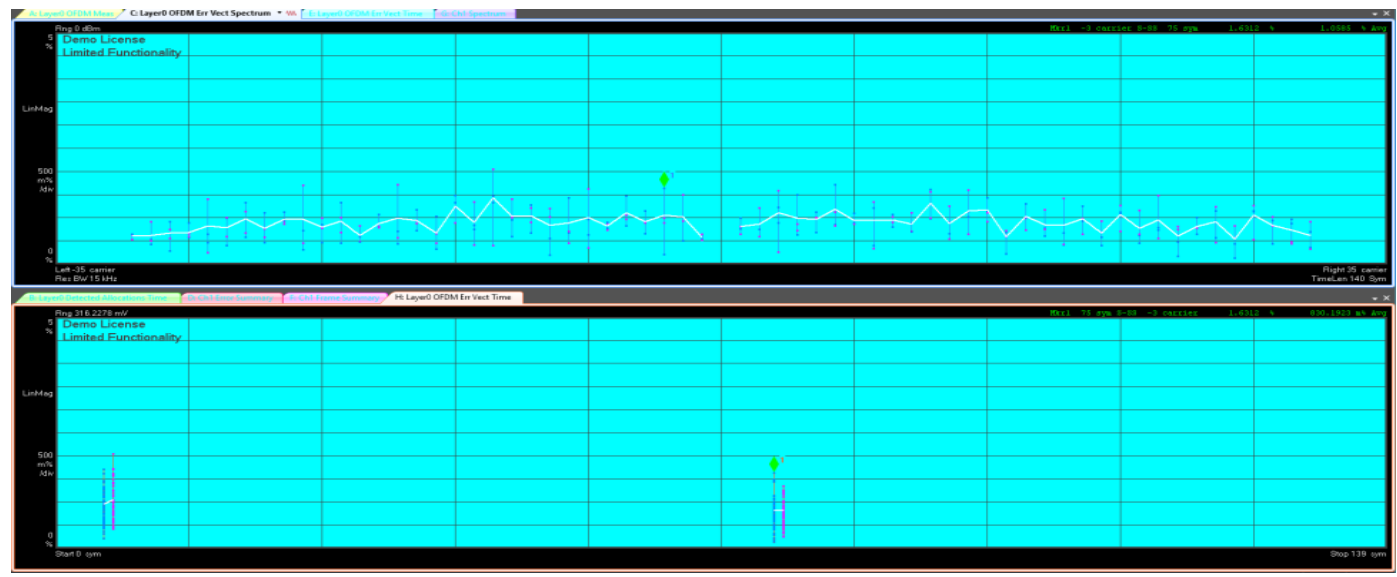

Figure 4d. Primary Synchronization Signal

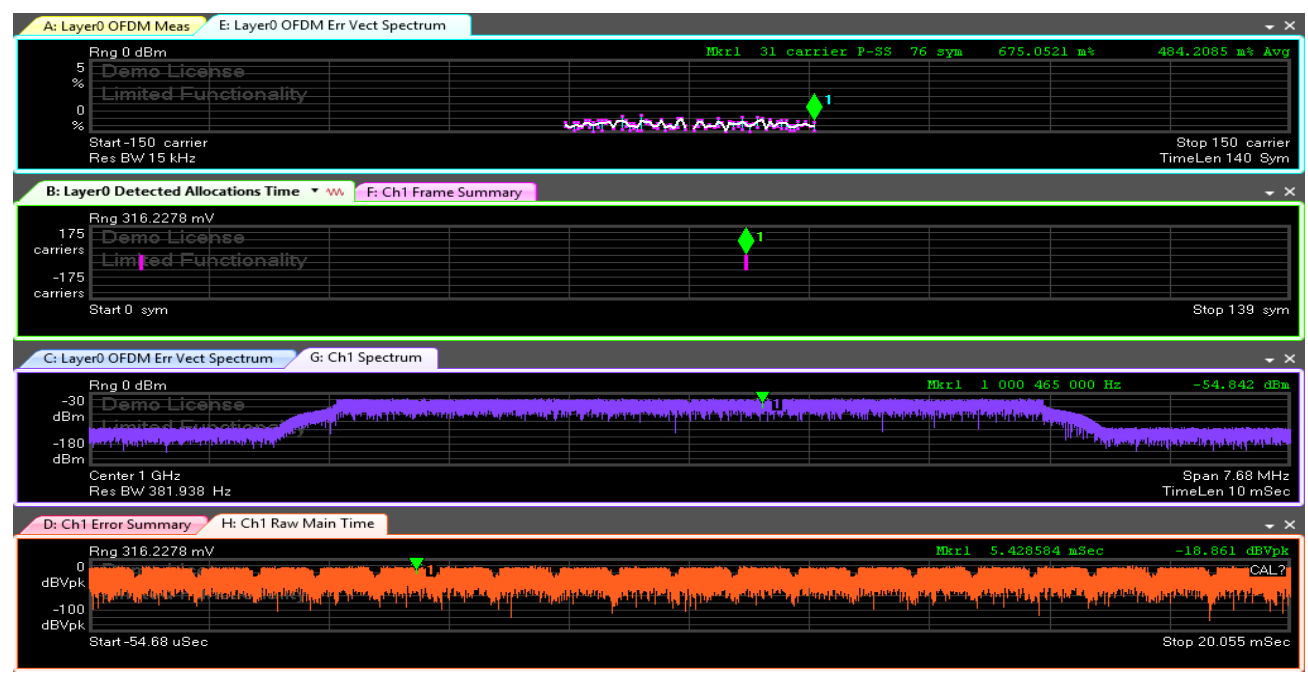

Figure 4e. Primary Synchronization Signal

Layer0_OFDM_Error_Vector_Spectrum: Only subcarrier indices are visible (i.e., -36 to 36). No actual value of subcarrier is known.Layer0_Detected_Allocations_Time: Symbol index can be known from this plot. But actual time value can't be known for any PBCH symbol. So, to avoid this problem, the following measurement traces are explored in VSA 89600B software.Ch1_Spectrum: The actual spectrum width is 1.08MHz.Ch1_Raw_Main_Time: The 1st PBCH Symbol occurs at 499.984131 microsec and the 4th PBCH Symbol occurs at 714.317464 microsec.

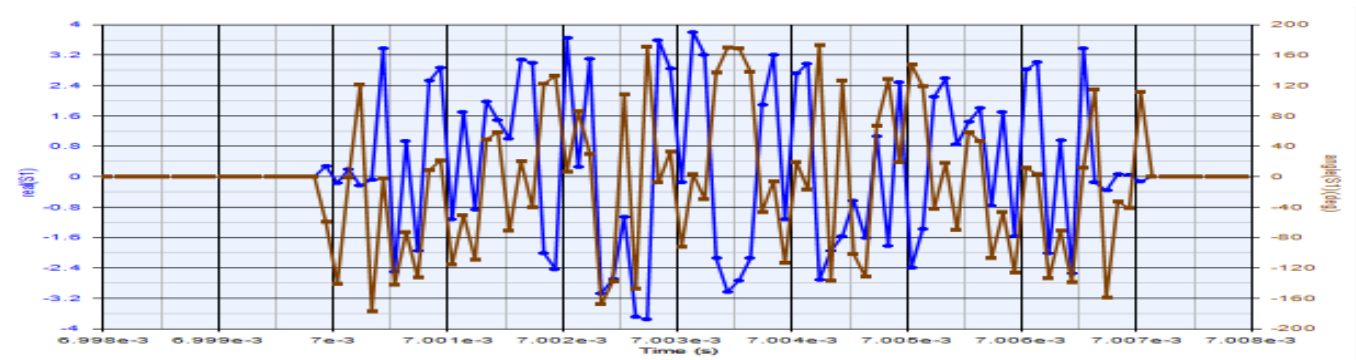

Figure 4f. P-SS mod Symbols with 1 of 3 Zadoff-Chu sequences (Blue - Real Part of the P-SS Mod symbols and Brown-Phase plot of the P-SS Mod symbols) 


\section{* Physical Broadcast Channel}

The below given is the Agilent Vector Signal Analyzer signal (VSA) for PBCH Channel

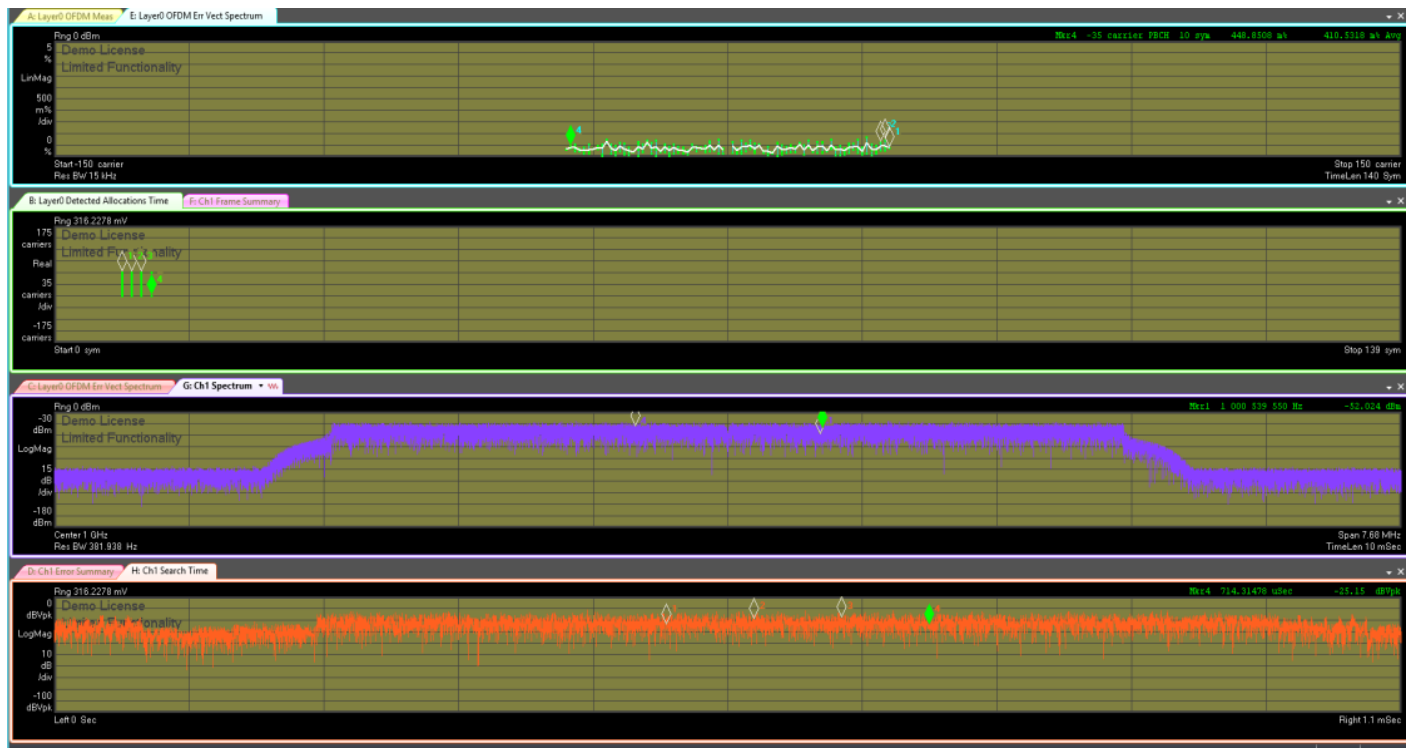

Figure 4g. PBCH Codes

Layer0_OFDM_Error_Vector_Spectrum: Only subcarrier indices are visible (i.e., -36 to 36). No actual value of subcarrier is known.Layer0_Detected_Allocations_Time: Symbol index can be known from this plot. But actual time value can't be known for any PBCH symbol. So, to avoid this problem, the following measurement traces are explored in VSA 89600B software.Ch1_Spectrum: The actual spectrum width is 1.08MHz.Ch1_Raw_Main_Time: The 1st PBCH Symbol occurs at 499.984131 microsec and the 4th PBCH Symbol occurs at 714.317464 microsec.

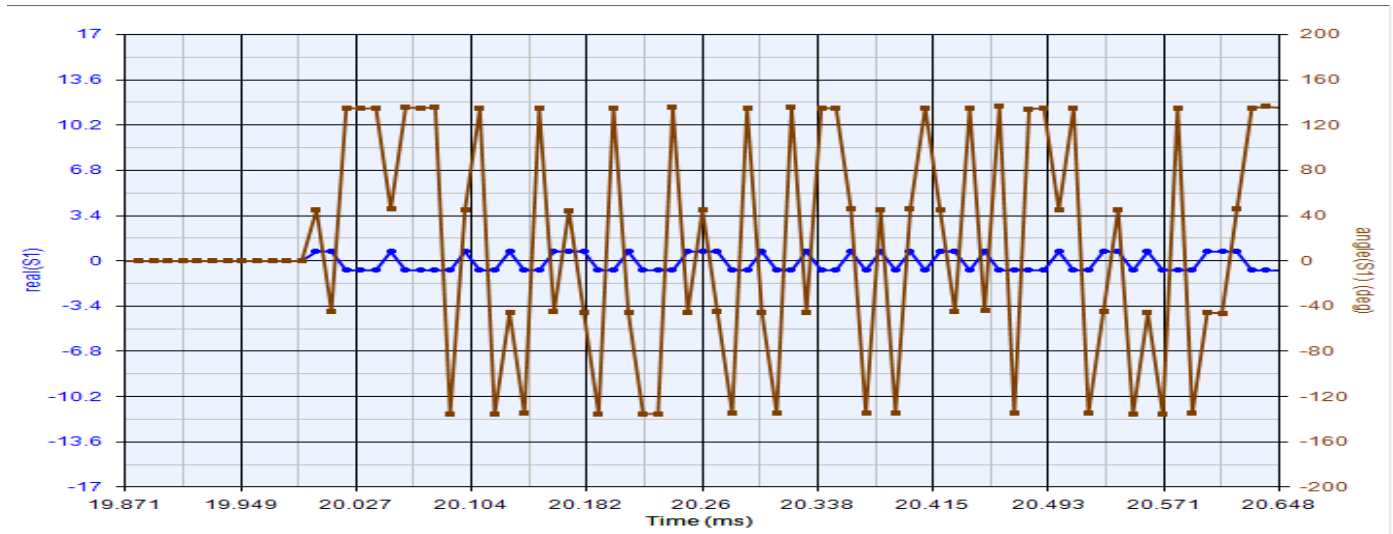

Figure 4h. PBCH mod Symbols with QPSK Modulation (Blue - Real Part of the PBCH Mod symbols and Brown-Phase plot of the PBCH Mod symbols)

\section{* Physical Control Format Indicator Channel}

The below given is the Agilent Vector Signal Analyzer signal (VSA) for PCFICH Channel 


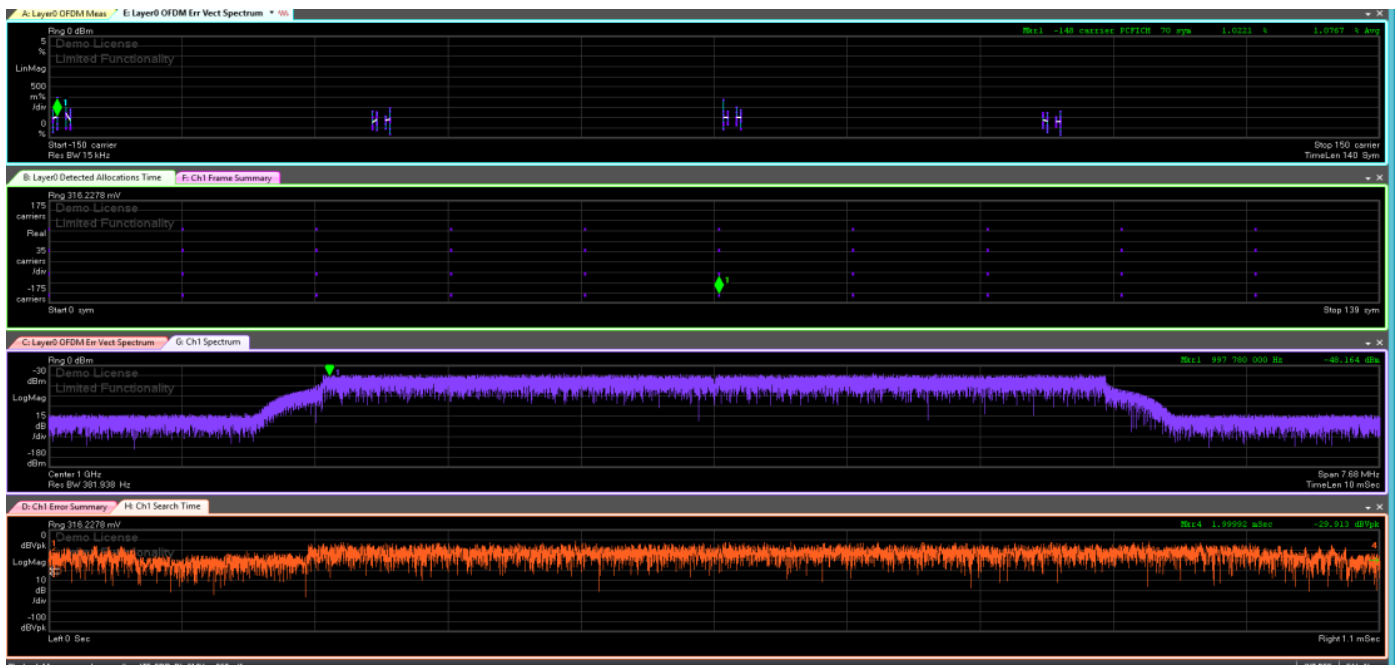

Figure 4i. PCFICH Codes

Layer0_OFDM_Error_Vector_Spectrum: Only subcarrier indices are visible.1st set of PCFICH subcarriers (belongs to -149 to -145). 2nd set of PCFICH subcarriers (belongs to -77 to -73 ). 3rd set of PCFICH subcarriers (belongs to 2 to 6).4th set of PCFICH subcarriers (belongs to 74 to 78). But No actual value of subcarrier is known.Layer0_Detected_Allocations_Time: Symbol index can be known from this plot. PCFICH symbols appear at symbol indices $(0,14,28,42,56,70,84,98,112,126)$. But actual time value can't be known for any PCFICH symbol. PCFICH symbol appears at starting index of each subframe. So, to avoid this problem, the following measurement traces are explored in VSA 89600B software.Ch1_Spectrum: The actual spectrum width is 3.405MHz.Ch1_Raw_Main_Time: The 1st PCFICH Symbol occurs at -15.869141 nanosec and the 10th PCFICH Symbol occurs at $8.999984 \mathrm{~ms}$.

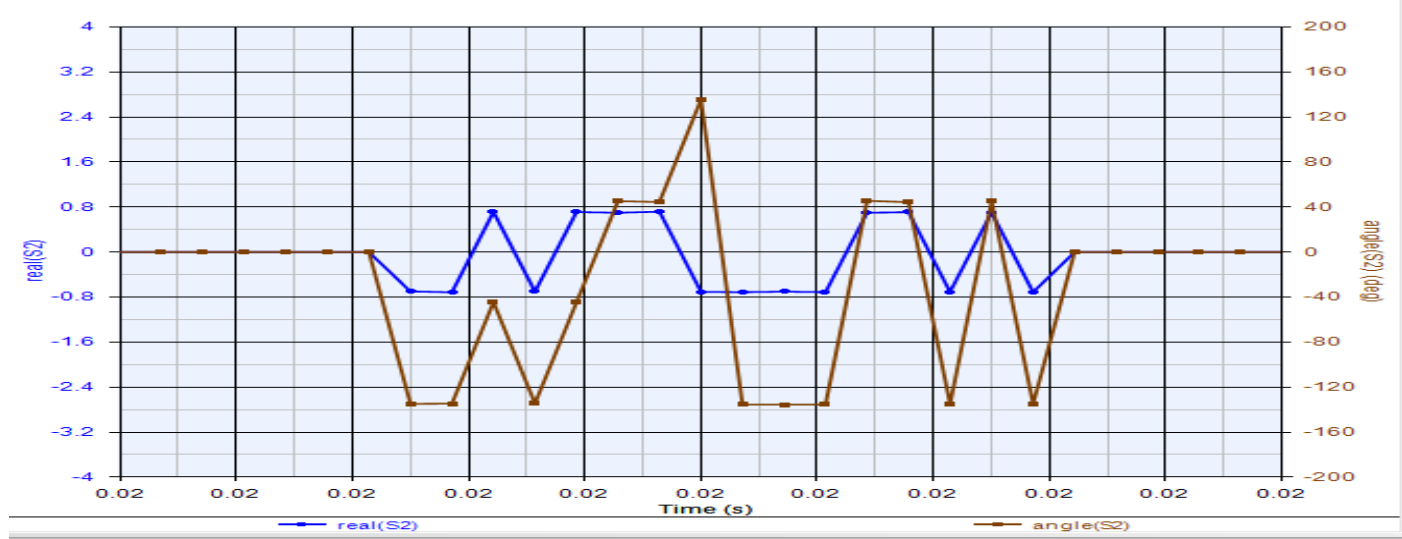

Figure 4j. PCFICH mod Symbols with QPSK Modulation (Blue - Real Part of the PCFICH Mod symbols and Brown-Phase plot of the PCFICH Mod symbols)

\section{* Physical Downlink Control Channel}

The below given is the Agilent Vector Signal Analyzer signal (VSA) for PDCCH Channel. 


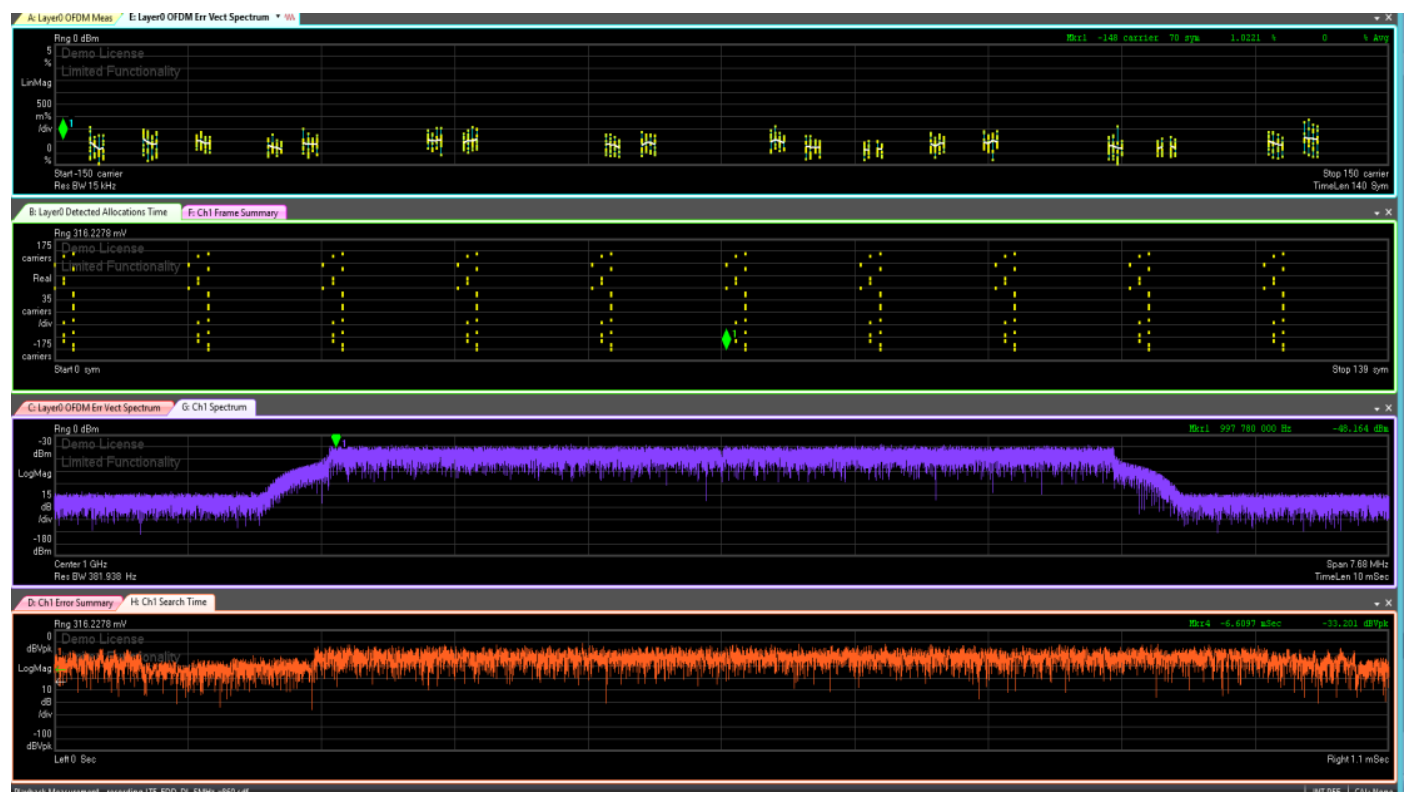

Figure 4k. Agilent VSA Output of PDCCH

The above Figure 4k shows the Agilent VSA Output of PDCCH.

Go to 'Markers' tab and select the 'Couple Markers'

Layer0_OFDM_Error_Vector_Spectrum: Only subcarrier indices are visible.

1st set of PDCCH subcarriers (belongs to -142 to -139 ).

2nd set of PDCCH subcarriers (belongs to -130 to -127 ).

3rd set of PDCCH subcarriers (belongs to -118 to -115 ).

4th set of PDCCH subcarriers (belongs to -102 to -99 ).

5th set of PDCCH subcarriers (belongs to -94 to -91 ).

6th set of PDCCH subcarriers (belongs to -66 to -63 ).

7 th set of PDCCH subcarriers (belongs to -58 to -55 ).

8th set of PDCCH subcarriers (belongs to -26 to -23 ).

12th set of PDCCH subcarriers (belongs to 32 to 36 ).

16th set of PDCCH subcarriers (belongs to 98 to 102).

17th set of PDCCH subcarriers (belongs to 123 to 126).

18th set of PDCCH subcarriers (belongs to 131 to 134).

But No actual value of subcarrier is known.Layer0_Detected_Allocations_Time: Symbol index can be known from this plot. PCFICH symbols appear at symbol indices $\{(0,1,2) \quad(14,15,16) \quad(28,29,30) \quad(42,43,44) \quad(56,57,58) \quad(70,71,72) \quad(84,85,86)$ $(98,99,100)(112,113,114)(126,127,128)\}$. But actual time value can't be known for any PDCCH symbol. PDCCH symbol appears at starting index of each subframe. So, to avoid this problem, the following measurement traces are explored in VSA 89600B software.Ch1_Spectrum: Each set of subcarriers occupies $45 \mathrm{KHz}$ bandwidth except 12 th \& 16th set of subcarriers. 12th \& 16th set of subcarriers are having individually a bandwidth of $60 \mathrm{KHz}$. The actual spectrum width is 16 x $45 \mathrm{KHz}+2 \times 60 \mathrm{KHz}=840$ KHz.

Ch1_Raw_Main_Time: The 1st PDCCH Symbol occurs at -15.869141 nanosec and the 30th PDCCH Symbol occurs at $9.142851 \mathrm{~ms}$. 


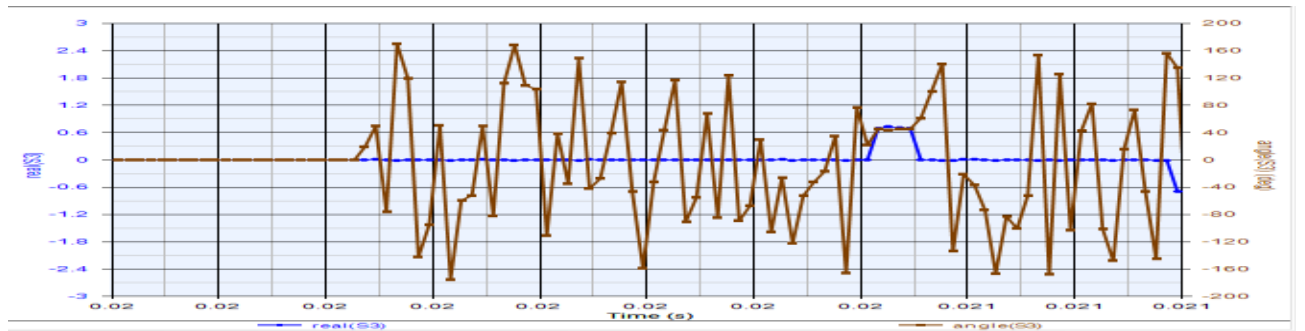

\section{Figure 4I. PDCCH mod Symbols with QPSK Modulation (Blue - Real Part of the PDCCH Mod symbols and Brown-Phase plot of the PDCCH Mod symbols)}

\section{Conclusion}

In this paper the Analysis of 4G LTE Physical Downlink Channel and the Physical signals is done using the Agilent Systemvue software. Signals which the downlink Channels carriers has been seen both in Frequency and time domain using the Agilent Vector Signal Analyzer (VSA).By testing the Signals at different levels like during design, testing at the production level, testing during repair even at the initial phase are of helpful in understanding the system. Therefore, for better understanding of $4 \mathrm{G} \_L T E$, this paper will enlighten Signal Measurement of downlink channels which helps to understand the DESIGN and SYSTHESIS of the 4G mobile system.

\section{References}

[1] S. S. A. Abbas, A. Kanimozhi and S. J. Thiruvengadam, "Realization of the SISO Architecture for PBCH of 3GPP-LTE using PlanAhead Tool and Virtex-5 Device", Department of Electronics and Communication Engineering, Mepco Schlenk Engineering College, Sivakasi, 626005, India, Department of Electronics and Communication Engineering, Thiagarajar College of Engineering, Madura, 625015, India, Journal of Wireless Networking and Communications, vol. 2, no. 3, (2012), pp. 14-22.

[2] M. Rumney, "LTE and the Evolution of 4G Wireless".

[3] I. Shah, S. Shukla, R. Shrotriya, N. Mehta, N. Mehta and S. Bakliwal, "Comparative Study of 4G Technology, Applications and Compatibility in Prevailing Networks", International Journal of Electronics Communication and Computer Technology (IJECCT), vol. 2, iss. 6, (2012).

[4] P. Gautam, S. Kaur, R. Kaur, S. Kaur and H. Kundra, "Review Paper on 4G Wireless Technology", MTech research scholar, Rayat Institute of Engineering and IT Railmajra, Associate Professor \& Head, Rayat Institute of Engineering and IT, Railmajra, International Journal of Advances in Science and Technology (IJAST), vol. 2, iss. 1, (2014).

[5] K. Kumaravel, "Comparative Study of 3G and 4G in Mobile Technology", Assistant Professor Dept. of Computer Science, Dr. N. G. P. Arts and Science College, Coimbatore, India , IJCSI International Journal of Computer Science Issues, vol. 8, iss. 5, no. 3, (2011).

[6] J. Parikh and A. Basu, "LTE Advanced: The 4G Mobile Broadband Technology", Bharati Vidyapeeth's College Of Engineering Delhi, India. International Journal of Computer Applications (0975 - 8887), vol. 13, no. 5, (2011).

[7] H. Thota, C. S Rani, K. Kagitha, A. Balagam, A. Goriparthi and N. Lakshmi Devarapalli, "Performance analysis of OFDM for $4 \mathrm{G}$ wireless systems under various fading channels", B.Tech, Electronics and Communication Engineering, DMS SVH College of Engineering, Machilipatnam, India ,Professor, Electronics and Communication Engineering, DMS SVH College of Engineering, Machilipatnam, India, vol 3, iss. 1, (2015).

[8] Krishnan, "Review Paper on 4G Communication Network", Lokmanya Tilak College of Enginnering, Mumbai University, India, International Journal of Emerging Technology and Advanced Engineering, vol. 4, iss. 9, (2014).

[9] C. Malarvizi and V. Nithya, "Enhancing Cell Edge Performance in cellular Mobile System", University College of Engineering Thirukkuvalai, Anna University, India, International Journal of Future Generation Communication and Networking, vol. 8, (2015), pp. 49-58.

[10] S. Kumar, T. Agrawal and P. Singh, "A Future Communication Technology: 5G' Department of Electronics an Communication Engineering", G.L.A University, Mathura,India, International Journal of Generation Communication and Networking, vol. 9, no. 1, (2016), pp. 303-310.

[11] www.keysight.com 